\title{
Editorial
}

\section{LINE-1 Retrotransposition: Impact on Genome Stability and Diversity and Human Disease}

\author{
Nina Luning Prak and Abdelali Haoudi \\ Department of Microbiology and Molecular Cell Biology, Eastern Virginia Medical School, Norfolk, Virginia 23501, USA
}

Received 6 February 2006; Accepted 6 February 2006

Copyright (C) 2006 N. L. Prak and A. Haoudi. This is an open access article distributed under the Creative Commons Attribution License, which permits unrestricted use, distribution, and reproduction in any medium, provided the original work is properly cited.

When we started thinking about devoting an issue of the Journal of Biomedicine and Biotechnology to LINE-1s, we were not sure it would fly. LINE-1s are long interspersed elements that account for $17 \%$ of the mass of the human genome (1), but far fewer than $17 \%$ of geneticists work on them! Nevertheless, L1s have received considerable press lately, including a number of high-profile stories featuring retrotransposition in L1 transgenic mice, functional studies of L1, and the potential contributions of L1s to the transcriptome (213). Based on these exciting developments in the L1 field and the collective enthusiasm and expertise of the contributors to this edition, we are pleased to present this special L1 issue.

The LINE-1 element (long interspersed element, L1) is an autonomous retrotransposon that propagates in the genome via retrotransposition. During retrotransposition, L1 DNA is transcribed to RNA and processed. The processed RNA is reverse-transcribed by the L1-encoded reverse transcriptase and the cDNA copy is inserted into a new chromosomal location. Over 500,000 L1 copies reside in the human genome, but L1 retrotransposition can also mobilize Alu elements (short interspersed elements) and contribute to processed pseudogene formation $(1,14-16)$. Much have been learned in recent years about L1 structure, function, and contribution to the genome, but much more remain to be understood, particularly how L1 insertions influence the expression and function of neighboring genes and how L1 mobility is kept in check.

This L1 issue is organized into two parts. The first part consists of six papers describing L1 biology (two papers) and the interactions of L1s with the genome (four papers). In a minireview, Sandra L Martin describes the structure and function of the L1 ORF1 protein during L1 retrotransposition. Her minireview article includes an update on recent studies describing L1 ORF1 protein-protein interactions, nu- cleic acid binding, and nucleic acid chaperone activity. In her paper, Mary F Lyon discusses the possible role of L1s in Xchromosome inactivation (XCI). Lyon presents evidence and a possible mechanism for the accumulation of L1s on the human X-chromosome in such a manner that they could fulfill the potential function as booster elements in XCI. Whether L1s are part of the mechanism of XCI or a result of it remains enigmatic. Shifting gears from L1 biology to L1 effects on the genome, Jian-Min Chen et al point to the challenges of detecting of autosomal L1-mediated insertions in their review article. In addition, Chen et al discuss the manner in which target genes are disrupted by L1-mediated retrotranspositional events and comment that these are likely to depend upon several different factors such as the type of insertion (ie, L1 direct, L1 trans-driven Alu, or L1 transdriven SVA), the precise locations of the inserted sequences within the target gene regions, the length of the inserted sequences, and possibly also their orientation. In their research article, Kert Matlik et al identify and characterize 49 chimeric L1 mRNAs, continuing the theme of L1 effects on genes. These chimeric transcripts are due to L1 sense or antisense promoter activity arising from within or nearby existing genes. In 45 out of the 49 cases, the chimeric transcript is in the same transcriptional orientation as the neighboring/surrounding gene. In addition, Matlik et al show that the L1 antisense promoter (ASP) can give rise to a chimeric transcript whose coding region is identical to the ORF of mRNA of several genes such as: KIAA1797, CLCN5, and SLCO1A2. Finally and most provocatively, they provide evidence that the L1 ASP can alter the tissue-specific pattern of transcription of some genes. Their study provides another dimension to the ways in which L1 can influence gene expression. In a minireview article, Sachiko Matsutani discusses the links between LINE-1 and SINE (Alu) elements and how L1-encoded 
proteins contribute to the mobilization of other mobile elements including Alu and processed pseudogenes and even cellular genes. In their minireview article, Todd Graham and Stephane Boissinot discuss factors affecting how L1s are distributed in the genome. L1 elements do not appear to be randomly distributed in the genome. Graham and Boissinot discuss factors that could skew the distribution of L1s in the genome, including L1 insertion bias and selection (either negative or positive) after insertion. The notion of negative selection arising when an L1 insertion has especially deleterious consequences (including increased recombination, altered transcription of neighboring genes, and continued retrotransposition) provides a bridge to the second section of the issue, which deals with the regulation of L1s.

The regulation of L1s can occur on many levels. L1 regulation can occur before the element has a chance to get going (pre- or posttranscriptional silencing, inefficient fulllength transcription due to premature polyadenylation, inefficient translation due to RNA editing) during retrotransposition (sequestration of L1 machinery in certain intracellular compartments, competition for L1 machinery by other substrates, blocking or modification of insertions by cellular DNA repair machinery) or after retrotransposition (apoptosis of a cell with a "disastrous" insertion, an immune response due to neoantigens created by the insertion or silencing of chromatin containing the insertion). This section on L1 regulation begins with a broad and learned overview by Wolfgang A Schulz. In this review article, Schulz describes why L1s might be afforded greater mobility in certain kinds of cancer cells and how changes in DNA repair machinery and epigenetic alterations could contribute to altered expression and activity of L1s. He critically reviews evidence for and against involvement of L1s in chromosomal breakage and recombination. In their research article, Kiyoshi Asada et al describe an animal model in which epigenetic alterations contribute to the development of hepatocellular carcinoma. Asada et al monitor the amount of cytosine methylation in the L1 5'UTR using combined bisulfite restriction analysis in rats fed a choline-deficient diet. They find that levels of L1 5'UTR methylation decrease with increasing age, with increasing length of time on the choline-deficient diet, and appear to be lower in tumor than in nontumor tissue. They discuss the potential utility of L1 methylation status as an indicator of genome-wide methylation and the potential contribution of altered L1 activity to genomic instability in tumors. The notion that L1s can contribute to genomic instability is further explored by Evan A Farkash who reviews the literature on mobile element activation and DNA damage. In his article review, Farkash describes ways in which L1s may be mobilized in the setting of genotoxic stress. Both of the final papers in this section focus on RNA interference (RNAi), an evolutionarily conserved process of sequence-specific, posttranscriptional gene silencing, as a potential mechanism for regulating L1s. Harris S Soifer reviews the published evidence of how RNAi controls mobile elements in other eukaryotes and provides a series of arguments for why RNAi would be a reasonable mechanism to constrain L1s in humans. In their review article, Shane R Horman et al describe how L1 RNA could be targeted by RNAi, with an emphasis on different forms of double-stranded and hairpin RNA. Horman et al also point out that the conventional cell-culture-based L1 retrotransposition assay (which relies on the expression of an antisense marker cassette) may induce RNAi.

We thank the contributors for their thought-provoking manuscripts and hope that readers will enjoy this special issue of the Journal of Biomedicine and Biotechnology on L1s.

Nina Luning Prak

Abdelali Haoudi

Nina Luning Prak is an Assistant Professor at the Department of Pathology and Laboratory Medicine at the University of Pennsylvania Health System. Nina Luning Prak conducted her PhD research on antibody gene rearrangement under the supervision of Martin Weigert. After completing medical school and a residency in clinical pathology at the hospital of the University of Pennsylvania, Nina joined the Laboratory of Haig Kazazian for a postdoctoral fellowship. With Members of the Kazazian Laboratory, Nina created an EGFP-tagged L1 transgenic mouse that her lab currently uses to study how L1 mobility is regulated. Of particular interest to the Luning Prak lab are how L1s mobilize in the setting of cell stress and whether RNAi limits L1 mobility. These and other topics pertaining to L1s and their ecology in the genome are highlighted in this special issue on L1 retrotransposons.

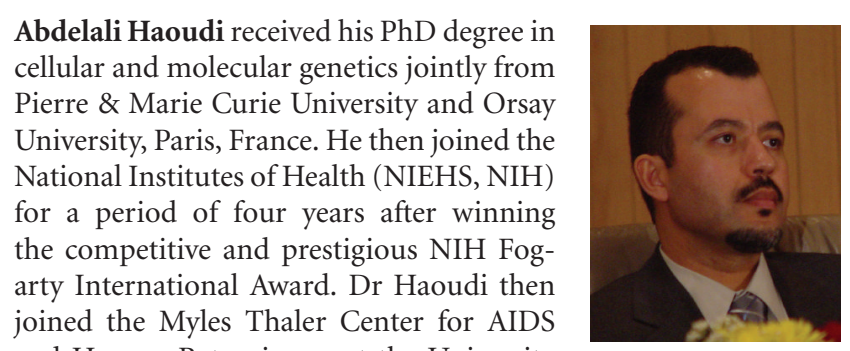
and Human Retroviruses at the University of Virginia Medical School, Charlottesville, then shortly after that, he joined the faculty at the Department of Microbiology and Molecular Cell Biology at Eastern Virginia Medical School in Norfolk, Va, in 2001. Dr Haoudi is interested in uncovering mechanisms by which mobile genetic retroelements, both retroviruses and retrotransposons, induce genetic instability and apoptosis in human cells and the molecular basis of cancer including cell cycle checkpoints and DNA repair mechanisms. Dr. Haoudi is also the Codirector of the Cancer Biology and Virology Focal Group. He has founded the Journal of Biomedicine and Biotechnology (http://www.j-biomed-biotech.org) and is also the Founder and President of the International Council of Biomedicine and Biotechnology (http://www.i-council-biomed-biotech.org). 

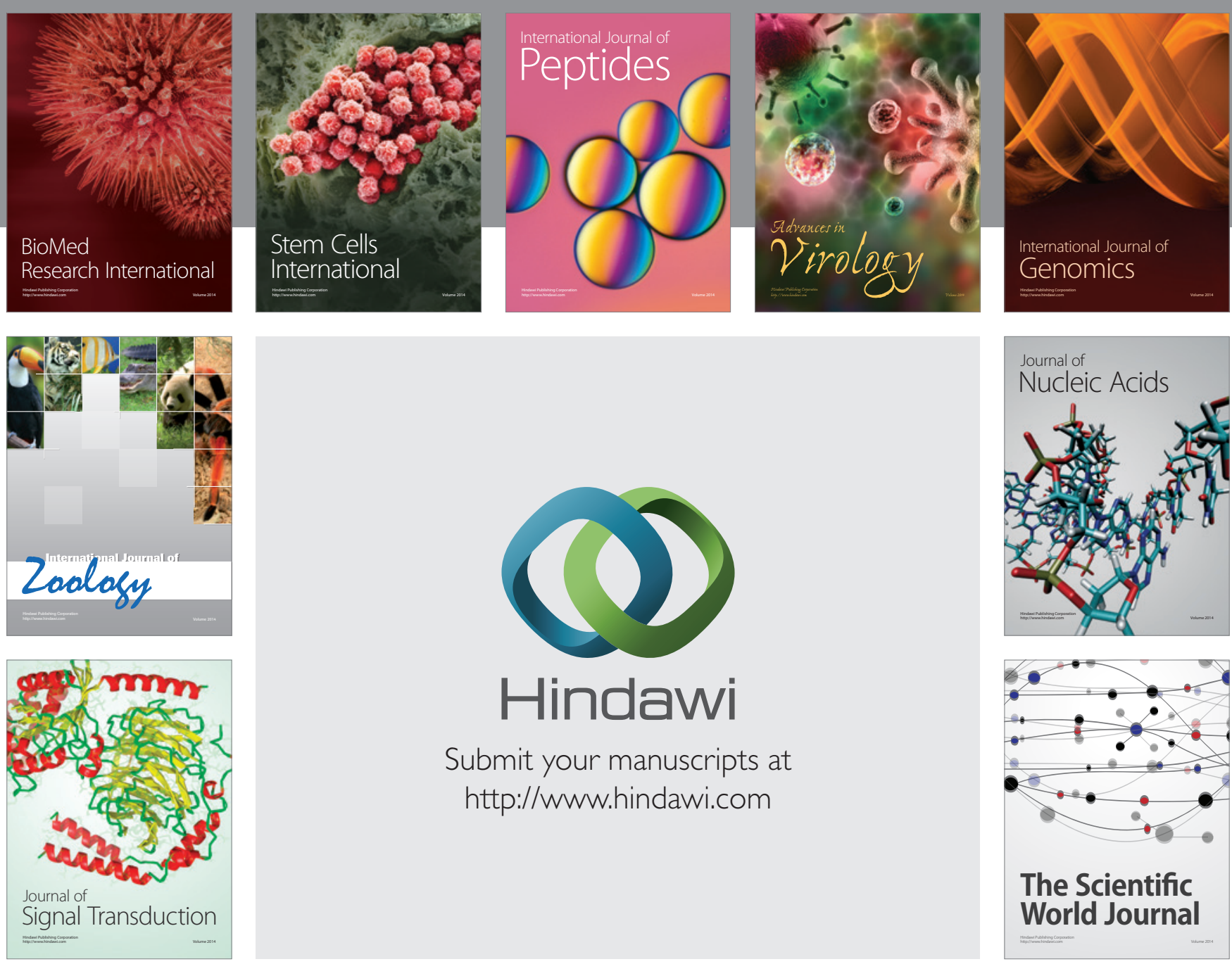

Submit your manuscripts at

http://www.hindawi.com
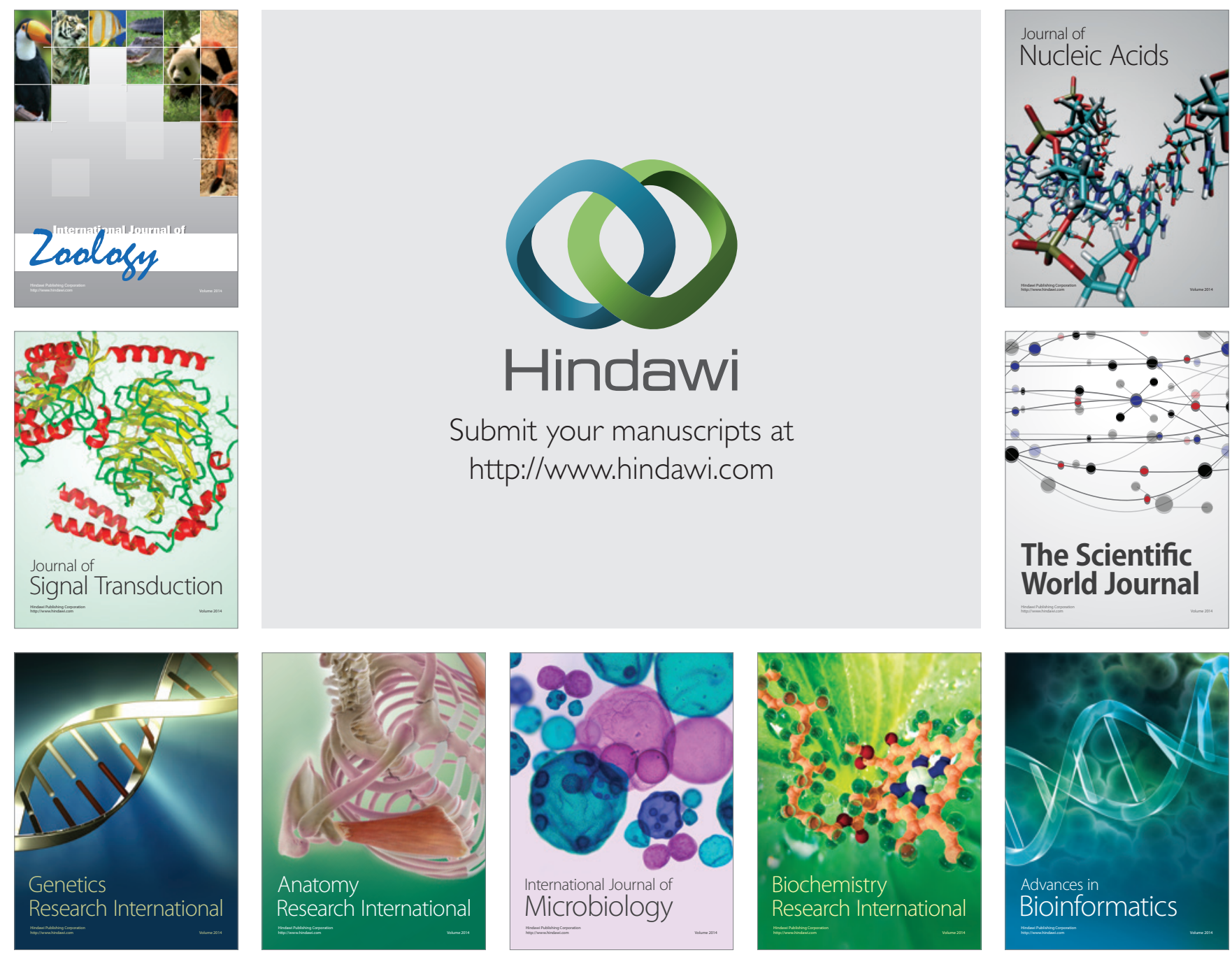

The Scientific World Journal
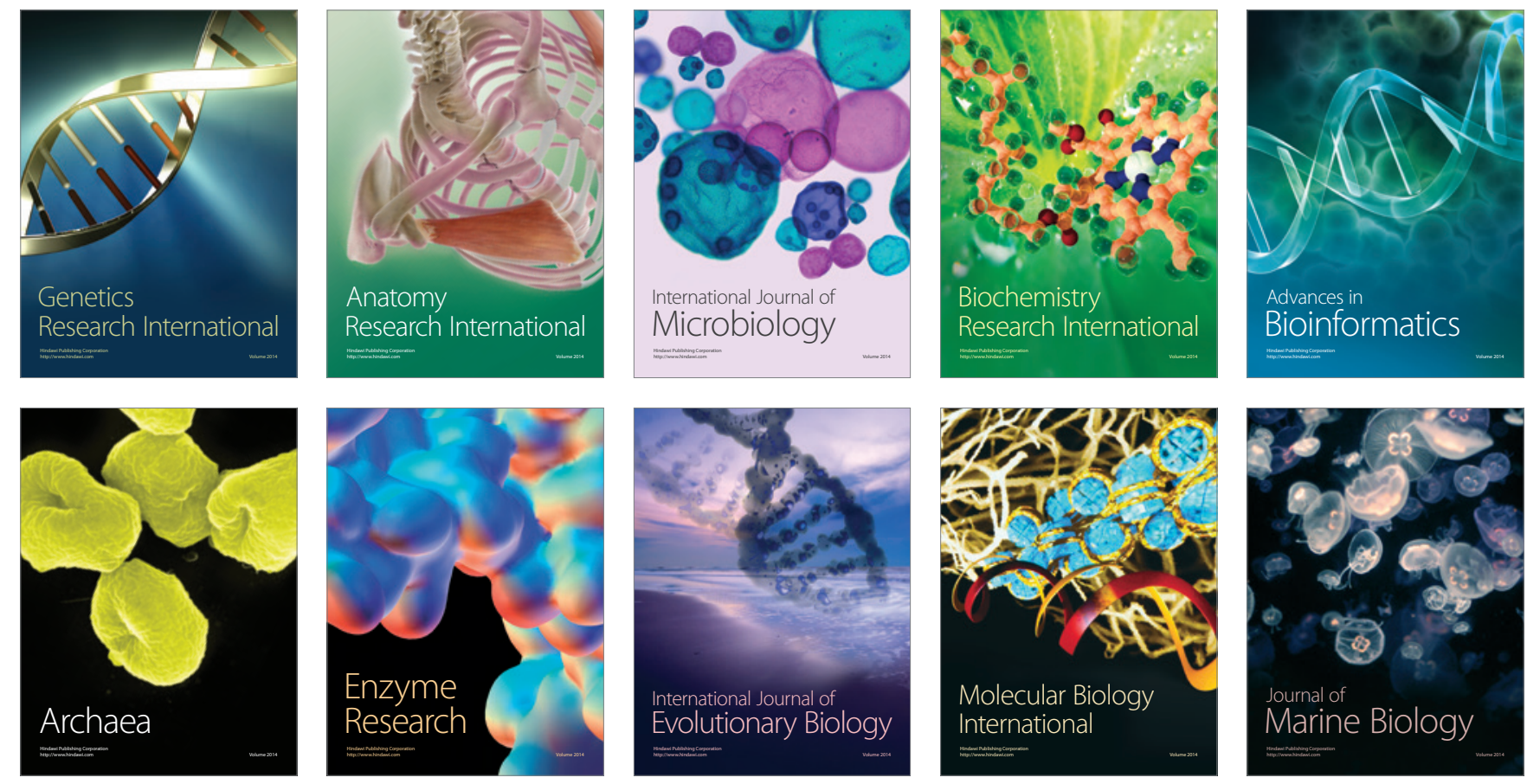